\title{
A WEIGHTED LEAST SQUARES FINITE ELEMENT METHOD FOR ELLIPTIC PROBLEMS WITH DEGENERATE AND SINGULAR COEFFICIENTS
}

\author{
S. BIDWELL, M. E. HASSELL, AND C. R. WESTPHAL
}

\begin{abstract}
We consider second order elliptic partial differential equations with coefficients that are singular or degenerate at an interior point of the domain. This paper presents formulation and analysis of a novel weighted-norm least squares finite element method for this class of problems. We propose a weighting scheme that eliminates the pollution effect and recovers optimal convergence rates. Theoretical results are carried out in appropriately weighted Sobolev spaces and include ellipticity bounds on the weighted homogeneous least squares functional, regularity bounds on the elliptic operator, and error estimates. Numerical experiments confirm the predicted error bounds.
\end{abstract}

\section{INTRODUCTION}

We consider partial differential equations of the form

$$
\left\{\begin{aligned}
-\nabla \cdot\left(r^{2 \beta} \nabla u\right)+r^{2 \alpha} u & =f & & \text { in } \Omega, \\
u & =0 & & \text { on } \partial \Omega,
\end{aligned}\right.
$$

where $\Omega \subset \mathbb{R}^{2}$ is an open connected convex polygonal domain with $\mathcal{O}=(0,0) \in \Omega$ and $r=\left(x^{2}+y^{2}\right)^{\frac{1}{2}}$ is the Euclidean distance from $\mathcal{O}$.

Problems of the type (1.1) generalize the well-studied class of strongly elliptic scalar equations by allowing coefficients which may degenerate (i.e., go to zero) or behave singularly (i.e., blow up) at the origin. Systems with such behavior include applications in quantum mechanics (see [18, 28]) and some reformulations of stochastic PDE (see e.g., 23] and references therein). Others have considered analysis of such problems in the context of a generalization of strongly elliptic equations (see e.g., 25, 24, 21). Problems similar to (1.1) arise, for example, when an elliptic problem with cylindrical or spherical symmetry is reduced to a lower dimensional problem (see e.g., [15, 22]).

Though for this paper we only consider operators with coefficients as in (1.1), it is straightforward to also consider coefficients that only locally behave as written, or cases with multiple points where coefficients are singular or degenerate. Assumptions on the smoothness of $\Omega$ are also for clarity of presentation, so that the only nonsmooth components of the solution are at $\mathcal{O}$ and not at boundary points.

\footnotetext{
Received by the editor August 31, 2010 and, in revised form, May 27, 2011 and October 5, 2011.

2010 Mathematics Subject Classification. Primary 65N30, 65N15, 35J70.

The research in this paper was supported by National Science Foundation Grant DMS-0755260.
} 
Compared with strongly elliptic equations, (1.1) will generally suffer a loss of regularity, making it especially difficult to obtain accurate numerical approximations. In general, numerical methods for problems with a loss of regularity may exhibit either a complete loss of convergence, or will converge slowly and with a pronounced pollution effect. In the latter case, the underlying metric that controls the error is unable to properly balance the error near singular points and error in regions of $\Omega$ where solutions are smooth. In [19, 20] and [13, least squares finite element formulations are equipped with weighted norms that induce a more optimal underlying metric on the error for problems with corner or edge singularities.

The least squares approach has additional benefits that motivate its use; for example, it simplifies the problem to a symmetric, positive definite linear system of equations, and it allows the freedom to choose finite element spaces for different unknowns. The least squares approach can also be used in conjunction with adaptive mesh refinement as the least squares method provides a natural error estimator for an adaptive routine. For primary background on least squares, refer to [11, 12, 5] and 14 .

In this paper, we develop a weighted-norm least squares finite element method for (1.1) that eliminates the pollution effect and yields optimal finite element convergence rates. We show that the homogeneous problem associated with (1.1) has a set of singular solutions when $\alpha=\beta-1$, and thus the focus of our analysis is on this case. We choose weight functions based on the expected form of the singular solutions, a simple calculation based on the coefficients of the original problem. The general approach we present need not be restricted to the $\alpha=\beta-1$ case, however, and numerical experiments in Section 4 confirm this. In 3, Arroyo, Bespalov and Heuer consider the standard variational formulation of (1.1) and prove error estimates for the numerical solution on graded meshes. Our approach achieves similar accuracy, but inherits the attractive features of a least squares discretization and does not require a graded mesh.

The organization of this paper is as follows. Section 2 defines notation and details the formulation of the weighted functional. In Section 3 we establish equivalence of the homogeneous weighted least squares functional to the appropriate norm and prove error bounds. Numerical experiments are given in Section 4 that demonstrate the effectiveness of the approach and support the theoretical results.

\section{Problem formulation}

We use standard notation for the $L^{2}$ norm, $\|\cdot\|$, and inner product, $\langle\cdot, \cdot \cdot$, and use $\|\cdot\|_{k ; \Omega}$ to denote the norm corresponding to the Sobolev space $H^{k}(\Omega)$, omitting subscript $\Omega$ when the domain is clear by context. For noninteger $k, H^{k}(\Omega)$ represents the standard interpolation space (see e.g., 2] ).

Define the weighted Sobolev norm by

$$
\|v\|_{k, w ; \Omega}=\left(\sum_{|j| \leq k}\left\|r^{w-k+|j|} D^{j} v\right\|_{0 ; \Omega}^{2}\right)^{1 / 2}
$$

and semi-norm by

$$
|v|_{k, w ; \Omega}=\left(\sum_{|j|=k}\left\|r^{w} D^{j} v\right\|_{0 ; \Omega}^{2}\right)^{1 / 2} .
$$


For $k=0,1$, we have $\|v\|_{0, w ; \Omega}=\left\|r^{w} v\right\|_{0 ; \Omega},\|v\|_{1, w ; \Omega}=\left(\left\|r^{w} \nabla v\right\|_{0 ; \Omega}^{2}+\left\|r^{w-1} v\right\|_{0 ; \Omega}^{2}\right)^{\frac{1}{2}}$ and $|v|_{1, w ; \Omega}=\left\|r^{w} \nabla v\right\|_{0 ; \Omega}$. The weighted Sobolev space $H_{w}^{k}(\Omega)$ is thus defined by

$$
H_{w}^{k}(\Omega)=\left\{v \in L^{2}(\Omega):\|v\|_{k, w ; \Omega}<+\infty\right\} .
$$

The dual spaces are defined by duality in the natural way. Let

$$
D_{w}^{1}=\left\{v \in H_{w}^{1}(\Omega): v=0 \text { on } \partial \Omega\right\},
$$

and define its dual, $H_{w}^{-1}(\Omega)$, by the norm

$$
\|v\|_{-1, w ; \Omega}=\sup _{\phi \in D_{-w}^{1}} \frac{|\langle v, \phi\rangle|}{\|\phi\|_{1,-w ; \Omega}} .
$$

By introducing the flux variable $\sigma=-r^{2 \beta} \nabla u$, we may expand system (1.1) to the first-order system,

$$
\left\{\begin{array}{rlrl}
\nabla \cdot \boldsymbol{\sigma}+r^{2 \alpha} u & =f & & \text { in } \Omega, \\
\boldsymbol{\sigma}+r^{2 \beta} \nabla u=0 & & \text { in } \Omega, \\
u & =0 & & \text { on } \partial \Omega .
\end{array}\right.
$$

The corresponding nonweighted functional is

$$
G(u, \boldsymbol{\sigma} ; f)=\left\|\nabla \cdot \boldsymbol{\sigma}+r^{2 \alpha} u-f\right\|^{2}+\left\|\boldsymbol{\sigma}+r^{2 \beta} \nabla u\right\|^{2},
$$

which yields less than optimal global discretization rates for these problems. Instead we consider the weighted least squares functional

$$
G_{w}(u, \boldsymbol{\sigma} ; f)=\left\|r^{w_{1}}\left(\nabla \cdot \boldsymbol{\sigma}+r^{2 \alpha} u-f\right)\right\|^{2}+\left\|r^{w_{2}}\left(\boldsymbol{\sigma}+r^{2 \beta} \nabla u\right)\right\|^{2},
$$

and investigate appropriate values for $w_{1}$ and $w_{2}$ to recover better rates of convergence and eliminate the pollution effect. Because convergence rates are based not only on the smoothness of the solution but also on the finite element space itself, we first consider the nullspace of (1.1).

We start by using asymptotic analysis to simplify the problem. Considering the homogeneous form of system (1.1),

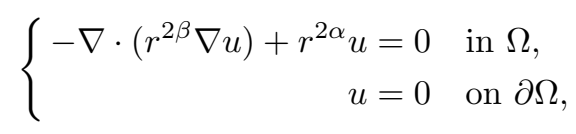

we look for singular solutions of the form $u=g(\theta) r^{\lambda}$. Using the polar form of the gradient,

$$
\nabla u=\frac{\partial u}{\partial r} \hat{r}+\frac{1}{r} \frac{\partial u}{\partial \theta} \hat{\theta}
$$

where $\hat{r}$ and $\hat{\theta}$ are unit vectors in the $r$ and $\theta$ directions, simple calculations reveal that

$$
\frac{g^{\prime \prime}+(2 \beta+\lambda) \lambda g}{g}=r^{2 \alpha-2 \beta+2} .
$$

Since the left side of equation (2.5) depends only on $\theta$, and the right side depends only on $r$, we obtain

$$
\begin{aligned}
2(\alpha-\beta+1) & =0, \\
g^{\prime \prime}+[(2 \beta+\lambda) \lambda-1] g & =0,
\end{aligned}
$$

which yields the restriction $\alpha=\beta-1$. 
Letting $\eta^{2}=(2 \beta+\lambda) \lambda-1$, we see that

$$
g=c_{1} \cos (\eta \theta)+c_{2} \sin (\eta \theta) \text { for } \eta=0,1,2, \ldots
$$

This restriction on $\eta$ gives

$$
\lambda_{n}=-\beta \pm \sqrt{\beta^{2}+\left(1+n^{2}\right)} \text { for } n=0,1,2, \ldots
$$

By invoking the boundary condition of $u=0$, we arrive at families of singular solutions in the nullspace of (2.4) of the form:

$$
u_{n}=\left(r^{-\beta+\sqrt{\beta^{2}+\left(1+n^{2}\right)}}-r^{-\beta-\sqrt{\beta^{2}+\left(1+n^{2}\right)}}\right) g(\theta) .
$$

We thus see that locally there exist two families of solutions. The family associated with the positive square root in (2.9) contains functions in $H^{1}(\Omega)$ and the family with the negative square root contains functions that are not in $L^{2}(\Omega)$. What we may conclude from this is that for sufficiently smooth $f$ there is at most one solution to (1.1) in $H^{1}(\Omega)$ (however, solutions may not be in $H^{2}(\Omega)$ ). Hereafter, we generally consider solutions with local behavior of the form $r^{\lambda}$ for $\lambda=-\beta+\sqrt{\beta^{2}+1}>0$ and also take $\alpha=\beta-1$.

Now, given $\alpha$ and $\lambda$ in terms of just $\beta$, we proceed to choose the weights for the functional. It is natural to choose weights that make the terms inside the least squares functional have the same power singularity as the solution. Recall the weighted least squares functional, (2.3), with flux variable $\sigma=-r^{2 \beta} \nabla u$. If the solution has the form $u \sim r^{\lambda}$, then the second term of the functional will have the form

$$
r^{w_{2}} \boldsymbol{\sigma} \sim r^{w_{2}+2 \beta+\lambda-1}
$$

and the first term will look like

$$
r^{w_{1}} \nabla \cdot \boldsymbol{\sigma} \sim r^{w_{1}+2 \beta+\lambda-2} .
$$

Thus, since we want to make these terms match the smoothness of the solution, we need

$$
w_{1}+2 \beta+\lambda-2=\lambda \text { and } w_{2}+2 \beta+\lambda-1=\lambda,
$$

which is true for the choice of weights

$$
w_{1}=2-2 \beta \quad \text { and } \quad w_{2}=1-2 \beta .
$$

In computation we typically choose weights according to this hueristic, which requires only a simple calculation based on the coefficients of the original problem. Additionally, in Section 3 we show that this choice of weights for the functional induces an equivalent weighted norm in which the error is minimized at optimal rates.

Thus, the least squares functional we focus on is

$$
G_{w}(u, \boldsymbol{\sigma} ; f)=\left\|r^{w_{1}}\left(\nabla \cdot \boldsymbol{\sigma}+r^{2 \beta-2} u-f\right)\right\|^{2}+\left\|r^{w_{2}}\left(\boldsymbol{\sigma}+r^{2 \beta} \nabla u\right)\right\|^{2},
$$

with $w_{1}=2-2 \beta$ and $w_{2}=1-2 \beta$ and the associated minimization problem is then to find $(u, \sigma) \in \mathcal{V} \times \mathcal{W}$ such that

$$
G_{w}(u, \boldsymbol{\sigma} ; f)=\inf _{(v, \boldsymbol{\tau}) \in \mathcal{V} \times \mathcal{W}} G_{w}(v, \boldsymbol{\tau} ; f),
$$

where

$$
\begin{aligned}
\mathcal{V} & =\left\{v \in H_{1}^{1}(\Omega): v=0 \text { on } \partial \Omega\right\} \\
\mathcal{W} & =\left\{\boldsymbol{\tau}: r^{w_{2}} \boldsymbol{\tau} \in L^{2}(\Omega), r^{w_{1}} \nabla \cdot \boldsymbol{\tau} \in L^{2}(\Omega)\right\}
\end{aligned}
$$


This minimization problem directly leads to the following symmetric variational problem: find $(u, \boldsymbol{\sigma}) \in \mathcal{V} \times \mathcal{W}$ such that

$$
a((u, \boldsymbol{\sigma}),(v, \boldsymbol{\tau}))=F(v, \boldsymbol{\tau})
$$

for all $(v, \boldsymbol{\tau}) \in \mathcal{V} \times \mathcal{W}$, where $a(\cdot, \cdot)$ and $F(\cdot)$ are defined by

$$
\begin{aligned}
a((u, \boldsymbol{\sigma}),(v, \boldsymbol{\tau})):= & \left\langle r^{2 w_{1}}\left(\nabla \cdot \boldsymbol{\sigma}+r^{2 \beta-2} u\right), \nabla \cdot \boldsymbol{\tau}+r^{2 \beta-2} v\right\rangle \\
& +\left\langle r^{2 w_{2}}\left(\boldsymbol{\sigma}+r^{2 \beta} \nabla u\right), \boldsymbol{\tau}+r^{2 \beta} \nabla v\right\rangle
\end{aligned}
$$

and

$$
F(v, \boldsymbol{\tau}):=\left\langle f, r^{w_{1}}\left(\nabla \cdot \boldsymbol{\tau}+r^{2 \beta-2} v\right)\right\rangle .
$$

Let $\mathcal{T}_{h}$ be a regular triangulation of $\Omega$ with a meshsize of $O(h)$, where on element $K$ we denote $h_{K}=\operatorname{diam}(K)$. Denote by $P_{k}(K)$ the standard space of polynomials of degree $\leq k$ on element $K$, and consider the Raviart-Thomas space of degree $k$ on element $K$ defined by

$$
R T_{k}(K)=P_{k}(K)+\mathbf{x} P_{k}(K),
$$

where $\mathbf{x}=(x, y)^{T}$. For this paper we define the spaces

$$
\begin{aligned}
\mathcal{V}^{h} & =\left\{v \in \mathcal{V} \cap C_{0}(\Omega):\left.v\right|_{K} \in P_{1}(K) \forall K \in \mathcal{T}_{h}\right\}, \\
\mathcal{W}^{h} & =\left\{\boldsymbol{\tau} \in \mathcal{W}:\left.\boldsymbol{\tau}\right|_{K} \in R T_{0}(K) \forall K \in \mathcal{T}_{h}\right\} .
\end{aligned}
$$

The discrete minimization problem is to find $\left(u^{h}, \sigma^{h}\right) \in \mathcal{V}^{h} \times \mathcal{W}^{h}$ such that

$$
G_{w}\left(u^{h}, \boldsymbol{\sigma}^{h} ; f\right)=\min _{\left(v^{h}, \boldsymbol{\tau}^{h}\right) \in \mathcal{V}^{h} \times \mathcal{W}^{h}} G_{w}\left(v^{h}, \boldsymbol{\tau}^{h} ; f\right) .
$$

This leads to a symmetric, positive definite linear system of equations that can be efficiently solved by a wide range of solvers. In particular, multigrid methods generally provide a robust solver for least squares discretizations. Though not the focus of this paper, it is important to remember that a successful numerical method should include an efficient solver for the resulting algebraic system. For expanded studies on the connection between least squares discretizations and multigrid solvers see [26, 8, 27, or [16] for an overview of the foundations connecting finite element discretizations to the associated linear system.

\section{Theoretical Results}

A general regularity result for strongly elliptic operators is established in 4, which we use to establish a weighted regularity bound for (1.1) for the case $\alpha=\beta-1$. We first consider the following $H_{w}^{1}$ estimate for strongly elliptic operators.

Theorem 3.1. Let $\mathcal{P}$ be a strongly elliptic operator with smooth coefficients where $\mathcal{P} u \in H_{w}^{-1}(\Omega)$ for $u \in \mathcal{V}$. Then for any $w \in \mathbb{R}$

$$
\|u\|_{1, w} \leq C\left(\|\mathcal{P} u\|_{-1, w}+\|u\|_{0, w-1}\right) .
$$

Proof. Theorem 3.7 in [4] proves this result with an additional trace term on the right side. Since we consider $u \in \mathcal{V}$, the trace term vanishes and the result follows directly.

We now consider a similar bound for our operator.

Theorem 3.2. Let $\mathcal{L} u:=-\nabla \cdot\left(r^{2 \beta} \nabla u\right)+r^{2 \beta-2} u$. For $u \in \mathcal{V}$ there is some $C>0$ such that

$$
\|u\|_{1,1} \leq C\left(\left\|r^{1-2 \beta} \mathcal{L} u\right\|_{-1,0}+\|u\|\right) .
$$


Proof. Note that for $p>0,\|x+y\|^{2} \geq(1-p)\|x\|^{2}-\left(\frac{1-p}{p}\right)\|y\|^{2}$, which, with $p=\frac{2}{3}$ and the facts $\nabla(r u)=r \nabla u+(\nabla r) u$ and $\|(\nabla r) u\|=\|u\|$, we have

$$
\begin{aligned}
\|r u\|_{1,0}^{2} & =\|\nabla(r u)\|^{2}+\|u\|^{2} \\
& =\|r \nabla u+(\nabla r) u\|^{2}+\|u\|^{2} \\
& \geq \frac{1}{3}\|r \nabla u\|^{2}-\frac{1}{2}\|(\nabla r) u\|^{2}+\|u\|^{2} \\
& \geq \frac{1}{3}\|r \nabla u\|^{2}+\frac{1}{2}\|u\|^{2} \\
& \geq \frac{1}{3}\|u\|_{1,1}^{2} .
\end{aligned}
$$

Now for any $\lambda_{1}, \lambda_{2}$, a straightforward calculation yields the identity

$$
r^{\lambda_{1}} \nabla \cdot\left(r^{\lambda_{2}} \nabla u\right)=\Delta\left(r^{\lambda_{1}+\lambda_{2}} u\right)-\left(\nabla r^{\lambda_{1}+\lambda_{2}}+r^{\lambda_{2}} \nabla r^{\lambda_{1}}\right) \cdot \nabla u-\Delta r^{\lambda_{1}+\lambda_{2}} u,
$$

a decomposition that can be found in [3. From this identity and a few simple calculations, we write $r^{1-2 \beta} \mathcal{L} u=-\Delta(r u)+\mathcal{L}_{1} u$, where $\mathcal{L}_{1} u:=(2-2 \beta) \nabla u \cdot \nabla r+$ $2 r^{-1} u$, which allows us to consider

$$
-\Delta(r u)=r^{1-2 \beta} \mathcal{L} u-\mathcal{L}_{1} u .
$$

Applying Theorem 3.1 with $w=0$, along with the triangle inequality, thus gives

$$
\begin{aligned}
\|r u\|_{1,0} & \leq C\left(\|-\Delta(r u)\|_{-1,0}+\|r u\|_{0,-1}\right) \\
& =C\left(\left\|r^{1-2 \beta} \mathcal{L} u+\mathcal{L}_{1} u\right\|_{-1,0}+\|u\|\right) \\
& \leq C\left(\left\|r^{1-2 \beta} f\right\|_{-1,0}+\|(2-2 \beta) \nabla r \cdot \nabla u\|_{-1,0}+\left\|2 r^{-1} u\right\|_{-1,0}+\|u\|\right) .
\end{aligned}
$$

We now bound the second and third terms on the right-hand side of (3.3). For the second term, using the definition of the weighted dual space, we have

$$
\begin{aligned}
\|(2-2 \beta) \nabla u \cdot \nabla r\|_{-1,0} \leq C\|\nabla u\|_{-1,0} & =C \sup _{\phi \in H_{0}^{1}} \frac{|\langle\nabla u, \phi\rangle|}{\|\phi\|_{1,0}} \\
& =C \sup _{\phi \in H_{0}^{1}} \frac{|\langle u, \nabla \cdot \phi\rangle|}{\left(\|\nabla \phi\|^{2}+\left\|r^{-1} \phi\right\|^{2}\right)^{\frac{1}{2}}} \\
& \leq C\|u\| .
\end{aligned}
$$

Similarly for the third term,

$$
\begin{aligned}
\left\|2 r^{-1} u\right\|_{-1,0} & \leq C \sup _{\phi \in H_{0}^{1}} \frac{\left|\left\langle r^{-1} u, \phi\right\rangle\right|}{\|\phi\|_{1,0}} \\
& =C \sup _{\phi \in H_{0}^{1}} \frac{\left|\left\langle u, r^{-1} \phi\right\rangle\right|}{\left(\|\nabla \phi\|^{2}+\left\|r^{-1} \phi\right\|^{2}\right)^{\frac{1}{2}}} \\
& \leq C\|u\| .
\end{aligned}
$$


Thus, from (3.3), combined with the bounds in (3.4) and (3.5), we have

$$
\|r u\|_{1,0} \leq C\left(\left\|r^{1-2 \beta} \mathcal{L} u\right\|_{-1,0}+\|u\|\right),
$$

and together with (3.1), this gives the desired result.

The following results establish the equivalence of $G_{w}(u, \sigma ; 0)^{\frac{1}{2}}$ to the norm

$$
\|(u, \boldsymbol{\sigma})\|_{1, w_{1}, w_{2}}:=\left(\|u\|_{1,1}^{2}+\|\boldsymbol{\sigma}\|_{0, w_{2}}^{2}+\|\nabla \cdot \boldsymbol{\sigma}\|_{0, w_{1}}^{2}\right)^{\frac{1}{2}}
$$

for $w_{2}=1-2 \beta, w_{1}=2-2 \beta$ and all $(u, \boldsymbol{\sigma}) \in \mathcal{V} \times \mathcal{W}$. Since $G_{w}\left(u^{h}, \boldsymbol{\sigma}^{h} ; f\right)=$ $G_{w}\left(u-u^{h}, \boldsymbol{\sigma}-\boldsymbol{\sigma}^{h} ; 0\right)$, this equivalence implies that minimizing the functional is equivalent to minimizing the error in this norm.

Theorem 3.3. For $w_{1}=w_{2}+1=2-2 \beta$ there exists a positive constant $C$ such that

$$
C\|(u, \boldsymbol{\sigma})\|_{1, w_{1}, w_{2}} \leq G_{w}(u, \boldsymbol{\sigma} ; 0)^{\frac{1}{2}}+\|u\|
$$

for all $(u, \sigma) \in \mathcal{V} \times \mathcal{W}$.

Proof. We first recall the definition of the homogeneous functional

$$
G_{w}(u, \boldsymbol{\sigma} ; 0)=\left\|r^{w_{1}}\left(\nabla \cdot \boldsymbol{\sigma}+r^{2 \beta-2} u\right)\right\|^{2}+\left\|r^{w_{2}}\left(\boldsymbol{\sigma}+r^{2 \beta} \nabla u\right)\right\|^{2} .
$$

Then Theorem 3.2 and the triangle inequality give

$$
\begin{aligned}
\|u\|_{1,1} & \leq C\left\|r^{1-2 \beta}\left(-\nabla \cdot\left(r^{2 \beta} \nabla u\right)+r^{2 \beta-2} u\right)\right\|_{-1,0}+c\|u\| \\
& \leq C\left\|r^{w_{2}} \nabla \cdot\left(r^{2 \beta} \nabla u+\boldsymbol{\sigma}\right)\right\|_{-1,0}+C\left\|r^{w_{2}}\left(\nabla \cdot \boldsymbol{\sigma}+r^{2 \beta-2} u\right)\right\|_{-1,0}+c\|u\| .
\end{aligned}
$$

We individually consider the first and second terms on the right side above. For the first term, integration by parts and the Cauchy-Schwarz inequality give

$$
\begin{aligned}
\left\|r^{w_{2}} \nabla \cdot\left(r^{2 \beta} \nabla u+\boldsymbol{\sigma}\right)\right\|_{-1,0} & =\sup _{\phi \in D_{0}^{1}} \frac{\mid\left\langle r^{2 \beta} \nabla u+\boldsymbol{\sigma}, \nabla\left(r^{w_{2}} \phi\right\rangle\right|}{\|\phi\|_{1,0}} \\
& =\sup _{\phi \in D_{0}^{1}} \frac{\left|\left\langle r^{w_{2}}\left(r^{2 \beta} \nabla u+\boldsymbol{\sigma}\right), \nabla \phi+\left(w_{2} \nabla r\right) r^{-1} \phi\right\rangle\right|}{\|\phi\|_{1,0}} \\
& \leq C\left\|r^{w_{2}}\left(r^{2 \beta} \nabla u+\boldsymbol{\sigma}\right)\right\| .
\end{aligned}
$$

The second term can be bounded similarly,

$$
\begin{aligned}
\left\|r^{w_{2}}\left(\nabla \cdot \boldsymbol{\sigma}+r^{2 \beta-2} u\right)\right\|_{-1,0} & =\sup _{\phi \in D_{0}^{1}} \frac{\left|\left\langle r^{w_{2}}\left(\nabla \cdot \boldsymbol{\sigma}+r^{2 \beta-2} u\right), \phi\right\rangle\right|}{\|\phi\|_{1,0}} \\
& =\sup _{\phi \in D_{0}^{1}} \frac{\left|\left\langle r^{w_{2}+1}\left(\nabla \cdot \boldsymbol{\sigma}+r^{2 \beta-2} u\right), r^{-1} \phi\right\rangle\right|}{\|\phi\|_{1,0}} \\
& \leq\left\|r^{w_{1}}\left(\nabla \cdot \boldsymbol{\sigma}+r^{2 \beta-2} u\right)\right\| .
\end{aligned}
$$

Combining equations (3.7), (3.8), and (3.9) gives

$$
\|u\|_{1,1} \leq C\left(G(u, \boldsymbol{\sigma} ; 0)^{\frac{1}{2}}+\|u\|\right) .
$$


It follows from the triangle inequality and $(3.10)$ that

$$
\begin{aligned}
& \|\boldsymbol{\sigma}\|_{0, w_{2}}+\|\nabla \cdot \boldsymbol{\sigma}\|_{0, w_{1}} \\
& \leq\left\|\boldsymbol{\sigma}+r^{2 \beta} \nabla u\right\|_{0, w_{2}} \\
& \quad+\left\|r^{2 \beta} \nabla u\right\|_{0, w_{2}}+\left\|\nabla \cdot \boldsymbol{\sigma}+r^{2 \beta-2} u\right\|_{0, w_{1}}+\left\|r^{2 \beta-2} u\right\|_{0, w_{1}} \\
& \leq G_{w}(u, \boldsymbol{\sigma} ; 0)^{\frac{1}{2}}+\|r \nabla u\|+\|u\| \\
& \leq G_{w}(u, \boldsymbol{\sigma} ; 0)^{\frac{1}{2}}+\|u\|_{1,1} \\
& \leq C\left(G_{w}(u, \boldsymbol{\sigma} ; 0)^{\frac{1}{2}}+\|u\|\right),
\end{aligned}
$$

which, together with (3.7), implies the desired result.

Theorem 3.4. If $w_{1}=w_{2}+1=2-2 \beta>0$, then there exists positive constants $c_{0}$ and $c_{1}$ such that

$$
c_{0}\|(u, \boldsymbol{\sigma})\|_{1, w_{1}, w_{2}} \leq G_{w}(u, \boldsymbol{\sigma} ; 0)^{\frac{1}{2}} \leq c_{1}\|(u, \boldsymbol{\sigma})\|_{1, w_{1}, w_{2}}
$$

for all $(u, \boldsymbol{\sigma}) \in \mathcal{V} \times \mathcal{W}$.

Proof. Using the triangle inequality, we have

$$
\begin{aligned}
G_{w}(u, \boldsymbol{\sigma} ; 0) & =\left\|r^{w_{1}}\left(\nabla \cdot \boldsymbol{\sigma}+r^{2 \beta-2} u\right)\right\|^{2}+\left\|r^{w_{2}}\left(\boldsymbol{\sigma}+r^{2 \beta} \nabla u\right)\right\|^{2} \\
& \leq C\left(\left\|r^{w_{1}} \nabla \cdot \boldsymbol{\sigma}\right\|^{2}+\left\|r^{w_{1}+2 \beta-2} u\right\|^{2}+\left\|r^{w_{2}} \boldsymbol{\sigma}\right\|^{2}+\left\|r^{w_{2}+2 \beta} \nabla u\right\|^{2}\right) \\
& =C\|(u, \boldsymbol{\sigma})\|_{1, w_{1}, w_{2}}^{2} .
\end{aligned}
$$

Taking the square root of both sides completes the upper bound. For the lower bound we recall the weak coercivity proved in Theorem 3.3 and use a modified compactness argument, a technique which is established in Lemma 3.3 and Theorem 3.4 in 20 . For this, we need only to establish that $H_{1}^{1}(\Omega)$ is compactly embedded in $H_{w_{1}}^{0}(\Omega)$ and that the operator induced by the functional is injective for $u \in \mathcal{V}$ to $H_{w_{1}}^{0}(\Omega)$. Chapter 6 of [17] establishes the compact imbedding, provided that $w_{1}>0$. (It is also interesting to note that $H_{1}^{1}(\Omega)$ is not compactly embedded in $H_{0}^{0}(\Omega)=L^{2}(\Omega)$, which necessitates the use of a modified compactness argument rather than a standard compactness argument.) To show injectivity we recall the asymptotic analysis in Section 2. Solutions to the homogeneous system (2.4) are of the form (2.9), which are not contained in $\mathcal{V}$. Since the operator is linear, we have that for problem (1.1), $f \in H_{w_{1}}^{0}(\Omega)$ implies a unique solution in $\mathcal{V}$. Thus, injectivity is established and the lower bound follows.

In what follows, we focus on numerical approximations, but we must first briefly recall several interpolation properties for $\mathcal{V}^{h} \times \mathcal{W}^{h}$. Let $\mathcal{I}^{h}$ be the standard interpolation operator onto $P_{k}(K)$, where, for all $v \in H^{m}(K)$,

$$
\left\|v-\mathcal{I}^{h} v\right\|_{s ; K} \leq C h_{K}^{m-s}|v|_{m ; K}
$$

holds for $0 \leq s \leq m$ and $1<m \leq k+1$. Details of this classical result can be found in [7] or [9]. 
Define the modified interpolation operator, $\mathcal{I}_{0}^{h}$, by

$$
\left.\mathcal{I}_{0}^{h} v\right|_{K}=\left\{\begin{aligned}
\left.\mathcal{I}^{h} v\right|_{K}, & \text { if } \bar{K} \cap \mathcal{O}=\emptyset, \\
\sum_{\substack{a_{i} \in K \\
a_{i} \neq \mathcal{O}}} v\left(a_{i}\right) \phi_{i}, & \text { if } \bar{K} \cap \mathcal{O} \neq \emptyset,
\end{aligned}\right.
$$

where $a_{i}$ are the nodal points corresponding to the basis functions, $\phi_{i}$. Thus, the modified interpolation has a value of zero at $\mathcal{O}$ and is identical to $\mathcal{I}^{h}$ away from $\mathcal{O}$. From [19, 20, if $w>0$, then $\mathcal{I}_{0}^{h}$ satisfies

$$
\sum_{K \in \mathcal{T}_{h}}\left\|v-\mathcal{I}_{0}^{h} v\right\|_{1, w ; K}^{2} \leq C h^{2}\|v\|_{2, w}^{2}
$$

for $v \in H_{w}^{2}(\Omega)$.

Let $I^{h}$ be the standard interpolation operator from $H($ div $) \cap L^{p}(\Omega)$ onto $R T_{k}$ with $p>2$. Then the following approximation properties of $R T_{k}$ hold (see, e.g., [10, 6] ) for any $\tau \in H^{m}(K)$ with $1 \leq m \leq k+1$,

$$
\left\|\boldsymbol{\tau}-I^{h} \boldsymbol{\tau}\right\|_{s ; K} \leq C h_{K}^{m-s}|\boldsymbol{\tau}|_{m ; K} \quad \text { with } \quad s=0,1,
$$

and

$$
\left\|\nabla \cdot\left(\boldsymbol{\tau}-I^{h} \boldsymbol{\tau}\right)\right\|_{0 ; K} \leq C h_{K}^{m}|\nabla \cdot \boldsymbol{\tau}|_{m ; K}
$$

if $\nabla \cdot \boldsymbol{\tau} \in H^{m}(K)$. For any $\boldsymbol{\tau}$ and $\nabla \cdot \boldsymbol{\tau}$ being only in $H^{\mu}(K)$ with $\mu<1$, we use the following approximation properties:

$$
\left\|\boldsymbol{\tau}-I^{h} \boldsymbol{\tau}\right\|_{0 ; K} \leq C h_{K}^{\mu}|\boldsymbol{\tau}|_{\mu ; K} \text { for } \mu>1 / 2
$$

and

$$
\left\|\nabla \cdot\left(\boldsymbol{\tau}-I^{h} \boldsymbol{\tau}\right)\right\|_{0 ; K} \leq C h_{K}^{\mu}|\nabla \cdot \boldsymbol{\tau}|_{\mu ; K} \text { for } \mu>0 .
$$

where in each case $C$ depends on $\mu$ and the shape of $K$. The bound in (3.17) follows by a direct appeal to the methods in the proof of Theorem 3.1 in [6], which corresponds to the case $\mu=1$. We omit the details for the sake brevity, but note that to accomplish this adaptation we must make use of the fractional seminorm definition

$$
|\boldsymbol{\tau}|_{\mu ; K}^{2}:=\int_{K} \int_{K} \frac{|\boldsymbol{\tau}(x)-\boldsymbol{\tau}(y)|^{2}}{|x-y|^{2+2 \mu}} d x d y
$$

and the corresponding trace theorem

$$
\|\boldsymbol{\tau}\|_{0 ; \partial K} \leq C\|\boldsymbol{\tau}\|_{\mu ; K} \text { for } \mu>1 / 2 .
$$

The bound in (3.18) follows by the well-known commutativity property $\nabla \cdot I^{h} \boldsymbol{\tau}=$ $\Pi^{h} \nabla \cdot \tau$, where $\Pi^{h}$ is the $L^{2}$-projection onto piecewise polynomials of degree less than or equal to $k-1$. Applying standard approximation properties of $\Pi^{h}$ confirms (3.18) for $\nabla \cdot \tau \in H^{\mu}(K)$.

For further details on interpolation in Raviart-Thomas spaces, refer to [1]. 
Theorem 3.5. Assume that $\boldsymbol{\tau}$ and $\nabla \cdot \boldsymbol{\tau}$ are in $H_{w}^{1}(\Omega) \cap H^{\mu}(\Omega)$. Then for any $w \geq 0$, there exists a positive constant $C$ such that

$$
\left\|\boldsymbol{\tau}-I^{h} \boldsymbol{\tau}\right\|_{0, w} \leq C h^{m}\left(|\boldsymbol{\tau}|_{1, w}+|\boldsymbol{\tau}|_{\mu}\right)
$$

and that

$$
\left\|\nabla \cdot\left(\boldsymbol{\tau}-I^{h} \boldsymbol{\tau}\right)\right\|_{0, w} \leq C h^{m}\left(|\nabla \cdot \boldsymbol{\tau}|_{1, w}+|\nabla \cdot \boldsymbol{\tau}|_{\mu}\right),
$$

where $m=\min \{1, w+\mu\}$ and $C$ depends only on $k, w$, and the shape of $\Omega$.

Proof. See Theorem 3.2 in [13].

We may now prove error bounds.

Theorem 3.6. Let $(u, \boldsymbol{\sigma})$ be the solution of system (2.1) where $u \in \mathcal{V} \cap H_{1}^{2}(\Omega)$, $\boldsymbol{\sigma} \in H_{w_{2}}^{1}(\Omega)^{2} \cap H^{\mu}(\Omega)^{2}$ and $\nabla \cdot \boldsymbol{\sigma} \in H_{w_{1}}^{1}(\Omega) \cap H^{\mu}(\Omega)$. If $\left(u^{h}, \boldsymbol{\sigma}^{h}\right)$ is the minimizer of the weighted functional (2.10) over $\mathcal{V}^{h} \times \mathcal{W}^{h}$ with $w_{1}=w_{2}+1=2-2 \beta$, then the following error estimate holds,

$$
\left\|\left(u-u^{h}, \boldsymbol{\sigma}-\boldsymbol{\sigma}^{h}\right)\right\|_{1, w_{1}, w_{2}} \leq C h^{m},
$$

where $m=\min \left\{1, w_{1}+\mu, w_{2}+\mu\right\}$.

Proof. For any $(v, \boldsymbol{\tau}) \in \mathcal{V}^{h} \times \mathcal{W}^{h}$, it follows from Theorem 3.4 and the orthogonality property that

$$
\begin{aligned}
c_{0}\left\|\left(u-u^{h}, \boldsymbol{\sigma}-\boldsymbol{\sigma}^{h}\right)\right\|_{1, w_{1}, w_{2}}^{2} \leq G\left(u-u^{h}, \boldsymbol{\sigma}-\boldsymbol{\sigma}^{h} ; 0\right) \\
=G(u-v, \boldsymbol{\sigma}-\boldsymbol{\tau} ; 0) \leq c_{1}\|(u-v, \boldsymbol{\sigma}-\boldsymbol{\tau})\|_{1, w_{1}, w_{2}}^{2},
\end{aligned}
$$

which implies

$$
\begin{aligned}
& \left\|\left(u-u^{h}, \boldsymbol{\sigma}-\boldsymbol{\sigma}^{h}\right)\right\|_{1, w_{1}, w_{2}}^{2} \leq \frac{c_{1}}{c_{0}} \min _{(v, \boldsymbol{\tau}) \in \mathcal{V}^{h} \times \mathcal{W}^{h}}\|(u-v, \boldsymbol{\sigma}-\boldsymbol{\tau})\|_{1, w_{1}, w_{2}}^{2} \\
& \quad \leq C\left(\inf _{v \in \mathcal{V}^{h}}\|u-v\|_{1,1}^{2}+\inf _{\boldsymbol{\tau} \in \mathcal{W}^{h}}\|\boldsymbol{\sigma}-\boldsymbol{\tau}\|_{0, w_{2}}^{2}+\inf _{\boldsymbol{\tau} \in \mathcal{W}^{h}}\|\nabla \cdot(\boldsymbol{\sigma}-\boldsymbol{\tau})\|_{0, w_{1}}^{2}\right) .
\end{aligned}
$$

This, with the approximation properties in (3.14), (3.19), and (3.20) results in $\left\|\left(u-u^{h}, \boldsymbol{\sigma}-\boldsymbol{\sigma}^{h}\right)\right\|_{1, w_{1}, w_{2}}^{2} \leq C h^{2 m}\left(\|u\|_{2,1}^{2}+|\boldsymbol{\sigma}|_{1, w_{2}}^{2}+|\boldsymbol{\sigma}|_{\mu}^{2}+|\nabla \cdot \boldsymbol{\sigma}|_{1, w_{1}}^{2}+|\nabla \cdot \boldsymbol{\sigma}|_{\mu}^{2}\right)$, which, under the smoothness assumptions on $u$ and $\sigma$, remains bounded and completes the proof.

\section{Numerical RESUlts}

In this section, we present some numerical experiments using the weighted-norm least squares approach to provide empirical evidence for the theoretical results provided in Section 3. For these examples, we select $\Omega=(-1,1) \times(-1,1)$ and choose $f$ so that $u=\left(1-x^{2}\right)\left(1-y^{2}\right) r^{\lambda}$, which satisfies the Dirichlet boundary condition on $\partial \Omega$ and allows locally nonsmooth behavior near the origin. For simplicity we compute all values on uniform triangulations of $\Omega$. We use $P_{1} \times R T_{0}$ finite element spaces for $\left(u^{h}, \boldsymbol{\sigma}^{h}\right)$, imposing Dirichlet boundary conditions on $u^{h}$ strongly.

In order to distinguish convergence rates over all of $\Omega$ from convergence rates near the origin, we define the following local region around the origin

$$
B_{\delta}=\{(x, y) \in \Omega: \max \{|x|,|y|\}<\delta\},
$$


which is simply a square centered at the origin. For all computations here we choose $\delta=0.4$. Notation for error measures are as follows: $G_{w}^{1 / 2}$ represents the weighted least squares functional, $\left\|u-u^{h}\right\|$ represents the global $L^{2}$ error between the exact and computed solutions over all of $\Omega$, and $\left\|u-u^{h}\right\|_{\Omega \backslash B_{\delta}}$ represents the $L^{2}$ norm over the domain with the singularity removed. Comparing these two $L^{2}$ norms is thus an indicator of the severity of the pollution effect. We denote the total number of elements by $N$, and convergence rates are computed with respect to $N^{-\frac{1}{2}}$.

For the first three examples we choose $\alpha=\beta-1$ and $\lambda=-\beta+\sqrt{\beta^{2}+1}$ as discussed in Section 2 Tables 4.1, 4.2 and 4.3 summarize results for $\beta=0.5, \beta=1$ and $\beta=1.25$, respectively, contrasting convergence behavior for a nonweighted least squares approach with the weighted approach as developed in this paper. In each case the nonweighted results show predictably disappointing results: slow convergence, even in regions away from the origin where the solution is smooth. But the weighted functional minimization is able to recover optimal convergence (i.e., the same as the interpolant), in regions with a nonsmooth solution as well as where the solution is smooth. Thus, the approach in this paper attenuates the pollution effect caused by the loss of regularity in solutions.

Nonweighted functional results

\begin{tabular}{|c|cc|cc|cc|}
\hline$N$ & $G^{1 / 2}$ & rate & $\left\|u-u^{h}\right\|$ & rate & $\left\|u-u^{h}\right\|_{\Omega \backslash B_{\delta}}$ & rate \\
\hline 1800 & 0.315 & - & $7.00 \mathrm{E}-03$ & - & $2.40 \mathrm{E}-04$ & - \\
5000 & 0.190 & 0.99 & $4.12 \mathrm{E}-03$ & 1.04 & $1.06 \mathrm{E}-03$ & 1.59 \\
9800 & 0.136 & 0.99 & $3.08 \mathrm{E}-03$ & 0.87 & $6.88 \mathrm{E}-04$ & 1.29 \\
16200 & 0.106 & 0.99 & $2.51 \mathrm{E}-03$ & 0.81 & $5.14 \mathrm{E}-04$ & 1.16 \\
24200 & 0.0867 & 0.99 & $2.14 \mathrm{E}-03$ & 0.79 & $4.11 \mathrm{E}-04$ & 1.11 \\
39200 & 0.0683 & 0.99 & $1.77 \mathrm{E}-03$ & 0.78 & $3.17 \mathrm{E}-04$ & 1.08 \\
57800 & 0.0563 & 0.99 & $1.52 \mathrm{E}-03$ & 0.78 & $2.57 \mathrm{E}-04$ & 1.07 \\
80000 & 0.0479 & 0.99 & $1.34 \mathrm{E}-03$ & 0.78 & $2.16 \mathrm{E}-04$ & 1.07 \\
\hline Optimal & - & 1 & - & $\approx 1.62$ & - & 2 \\
\hline
\end{tabular}

Weighted functional results, $w_{1}=1, w_{2}=0$

\begin{tabular}{|c|cc|cc|cc|}
\hline$N$ & $G_{w}^{1 / 2}$ & rate & $\left\|u-u^{h}\right\|$ & rate & $\left\|u-u^{h}\right\|_{\Omega \backslash B_{\delta}}$ & rate \\
\hline 1800 & 0.276 & - & $7.56 \mathrm{E}-03$ & - & $2.59 \mathrm{E}-03$ & - \\
5000 & 0.166 & 1.00 & $3.35 \mathrm{E}-03$ & 1.59 & $9.41 \mathrm{E}-04$ & 1.98 \\
9800 & 0.119 & 1.00 & $1.96 \mathrm{E}-03$ & 1.59 & $4.82 \mathrm{E}-04$ & 1.99 \\
16200 & 0.0924 & 1.00 & $1.32 \mathrm{E}-03$ & 1.59 & $2.92 \mathrm{E}-04$ & 1.99 \\
24200 & 0.0756 & 1.00 & $9.56 \mathrm{E}-04$ & 1.59 & $1.96 \mathrm{E}-04$ & 1.99 \\
39200 & 0.0594 & 1.00 & $6.51 \mathrm{E}-04$ & 1.59 & $1.21 \mathrm{E}-04$ & 1.99 \\
57800 & 0.0489 & 1.00 & $4.78 \mathrm{E}-04$ & 1.60 & $8.25 \mathrm{E}-05$ & 1.99 \\
80000 & 0.0416 & 1.00 & $3.68 \mathrm{E}-04$ & 1.60 & $5.97 \mathrm{E}-05$ & 1.99 \\
\hline Optimal & - & 1 & - & $\approx 1.62$ & - & 2 \\
\hline
\end{tabular}

TABLE 4.1. Convergence of approximations for nonweighted least squares functional vs. using a weighted functional for $\beta=0.5$, $\alpha=-0.5$ and $\lambda=0.62$. 
Non-weighted functional results

\begin{tabular}{|c|cc|cc|cc|}
\hline$N$ & $G^{1 / 2}$ & rate & $\left\|u-u^{h}\right\|$ & rate & $\left\|u-u^{h}\right\|_{\Omega \backslash B_{\delta}}$ & rate \\
\hline 1800 & 0.367 & - & $1.26 \mathrm{E}-02$ & - & $3.06 \mathrm{E}-03$ & - \\
5000 & 0.220 & 1.00 & $9.84 \mathrm{E}-03$ & 0.48 & $1.14 \mathrm{E}-03$ & 1.93 \\
9800 & 0.157 & 1.00 & $8.17 \mathrm{E}-03$ & 0.55 & $6.00 \mathrm{E}-04$ & 1.91 \\
16200 & 0.123 & 1.00 & $7.03 \mathrm{E}-03$ & 0.60 & $3.73 \mathrm{E}-04$ & 1.89 \\
24200 & 0.100 & 1.00 & $6.19 \mathrm{E}-03$ & 0.63 & $2.56 \mathrm{E}-04$ & 1.87 \\
39200 & 0.0788 & 1.00 & $5.30 \mathrm{E}-03$ & 0.65 & $1.64 \mathrm{E}-04$ & 1.86 \\
57800 & 0.0649 & 1.00 & $4.65 \mathrm{E}-03$ & 0.67 & $1.15 \mathrm{E}-04$ & 1.84 \\
80000 & 0.0551 & 1.00 & $4.17 \mathrm{E}-03$ & 0.68 & $8.54 \mathrm{E}-05$ & 1.82 \\
\hline Optimal & - & 1 & - & $\approx 1.41$ & - & 2 \\
\hline
\end{tabular}

Weighted functional results, $w_{1}=0, w_{2}=-1$

\begin{tabular}{|c|cc|cc|cc|}
\hline$N$ & $G_{w}^{1 / 2}$ & rate & $\left\|u-u^{h}\right\|$ & rate & $\left\|u-u^{h}\right\|_{\Omega \backslash B_{\delta}}$ & rate \\
\hline 1800 & 0.364 & - & $6.05 \mathrm{E}-03$ & - & $2.82 \mathrm{E}-03$ & - \\
5000 & 0.219 & 1.00 & $2.78 \mathrm{E}-03$ & 1.52 & $1.02 \mathrm{E}-03$ & 1.99 \\
9800 & 0.156 & 1.00 & $1.68 \mathrm{E}-03$ & 1.49 & $5.20 \mathrm{E}-04$ & 2.00 \\
16200 & 0.122 & 1.00 & $1.16 \mathrm{E}-03$ & 1.47 & $3.15 \mathrm{E}-04$ & 2.00 \\
24200 & 0.0995 & 1.00 & $8.68 \mathrm{E}-04$ & 1.46 & $2.11 \mathrm{E}-04$ & 2.00 \\
39200 & 0.0782 & 1.00 & $6.12 \mathrm{E}-04$ & 1.45 & $1.30 \mathrm{E}-04$ & 2.00 \\
57800 & 0.0644 & 1.00 & $4.62 \mathrm{E}-04$ & 1.44 & $8.83 \mathrm{E}-05$ & 2.00 \\
80000 & 0.0547 & 1.00 & $3.66 \mathrm{E}-04$ & 1.44 & $6.38 \mathrm{E}-05$ & 2.00 \\
\hline Optimal & - & 1 & - & $\approx 1.41$ & - & 2 \\
\hline
\end{tabular}

TABLE 4.2. Convergence of approximations for nonweighted least squares functional vs. using a weighted functional for $\beta=1, \alpha=0$ and $\lambda=0.41$.

In Table 4.4, we consider $\beta=0.5, \alpha=-0.25$, a case not covered by the theory developed in this paper since $\alpha \neq \beta-1$. For this problem we consider $\lambda=0.62$ and choose $w_{1}=1.0, w_{2}=0.5$ to achieve results similar to the other cases.

The final numerical result provides a more direct comparison to the approach by Arroyo, Bespalov and Heuer. For this example we consider Example B2 in [3] with parameters $\beta=0, \alpha=-1$, and $\lambda=0.5$. We choose weights $w_{1}=1.5, w_{2}=0.5$ based on this weak singularity. The range of mesh sizes are chosen from the same range as in B2 and we also compute the error with respect to the normalized $H_{0.6}^{1}(\Omega)$ norm,

$$
E_{h}=\frac{\left\|u-u^{h}\right\|_{1,0.6}}{\|u\|_{1,0.6}} .
$$

This is a norm with a strong enough weight to guarantee $O(h)$ convergence. Table 4.5 shows that our results have the expected $O(h)$ convergence with respect to the weighted functional norm as well as approximately $O(h)$ in the $E_{h}$ measure. Not only do our results have the same asymptotic behavior as the approach in [3], but the actual values for $E_{h}$ are in close agreement. It is also interesting to note that, as we may expect, $E_{h}$ and $G_{w}^{1 / 2}$ are good indicators of the convergence for such a problem, but $G_{w}^{1 / 2}$ may be used with confidence on more challenging problems where the exact solution is not known. 
Nonweighted functional results

\begin{tabular}{|c|cc|cc|cc|}
\hline$N$ & $G^{1 / 2}$ & rate & $\left\|u-u^{h}\right\|$ & rate & $\left\|u-u^{h}\right\|_{\Omega \backslash B_{\delta}}$ & rate \\
\hline 1800 & 0.419 & - & $3.87 \mathrm{E}-02$ & - & $3.39 \mathrm{E}-03$ & - \\
5000 & 0.252 & 1.00 & $3.18 \mathrm{E}-02$ & 0.39 & $1.27 \mathrm{E}-03$ & 1.93 \\
9800 & 0.180 & 1.00 & $2.69 \mathrm{E}-02$ & 0.50 & $6.62 \mathrm{E}-04$ & 1.92 \\
16200 & 0.140 & 1.00 & $2.34 \mathrm{E}-02$ & 0.55 & $4.09 \mathrm{E}-04$ & 1.92 \\
24200 & 0.115 & 1.00 & $2.09 \mathrm{E}-02$ & 0.58 & $2.78 \mathrm{E}-04$ & 1.91 \\
39200 & 0.0900 & 1.00 & $1.81 \mathrm{E}-02$ & 0.60 & $1.76 \mathrm{E}-04$ & 1.91 \\
57800 & 0.0741 & 1.00 & $1.60 \mathrm{E}-02$ & 0.62 & $1.21 \mathrm{E}-04$ & 1.91 \\
80000 & 0.0630 & 1.00 & $1.45 \mathrm{E}-02$ & 0.63 & $8.92 \mathrm{E}-05$ & 1.90 \\
\hline Optimal & - & 1 & - & $\approx 1.35$ & - & 2 \\
\hline
\end{tabular}

Weighted functional results, $w_{1}=-0.5, w_{2}=-1.5$

\begin{tabular}{|c|cc|cc|cc|}
\hline$N$ & $G_{w}^{1 / 2}$ & rate & $\left\|u-u^{h}\right\|$ & rate & $\left\|u-u^{h}\right\|_{\Omega \backslash B_{\delta}}$ & rate \\
\hline 1800 & 0.440 & - & $6.09 \mathrm{E}-03$ & - & $3.17 \mathrm{E}-03$ & - \\
5000 & 0.264 & 1.00 & $2.83 \mathrm{E}-03$ & 1.50 & $1.15 \mathrm{E}-03$ & 1.99 \\
9800 & 0.189 & 1.00 & $1.74 \mathrm{E}-03$ & 1.45 & $5.86 \mathrm{E}-04$ & 2.00 \\
16200 & 0.147 & 1.00 & $1.22 \mathrm{E}-03$ & 1.42 & $3.55 \mathrm{E}-04$ & 2.00 \\
24200 & 0.120 & 1.00 & $9.17 \mathrm{E}-04$ & 1.41 & $2.38 \mathrm{E}-04$ & 2.00 \\
39200 & 0.0945 & 1.00 & $6.56 \mathrm{E}-04$ & 1.39 & $1.47 \mathrm{E}-04$ & 2.00 \\
57800 & 0.0778 & 1.00 & $5.01 \mathrm{E}-04$ & 1.38 & $9.96 \mathrm{E}-05$ & 2.00 \\
80000 & 0.0661 & 1.00 & $4.01 \mathrm{E}-04$ & 1.38 & $7.20 \mathrm{E}-05$ & 2.00 \\
\hline Optimal & - & 1 & - & $\approx 1.35$ & - & 2 \\
\hline
\end{tabular}

TABLE 4.3. Convergence of approximations for nonweighted least squares functional vs. using a weighted functional for $\beta=1.25$, $\alpha=0.25$ and $\lambda=0.35$.

In summary, our numerical experiments confirm the theory developed in this paper and show that we may achieve analogous results to other finite element approaches. The weighted norm approach developed here inherits many advantages of the least squares approach. The linear systems are symmetric positive definite and are generally easy to solve with standard iterative methods. There is no need for graded meshes or enhancing the finite element spaces; instead, the weighted norm itself defines an appropriate metric to balance the error across the domain which eliminates the pollution effect. In addition, the weighted functional provides a natural and free error measure in an appropriate weighted norm. We have shown that the severity of the singular solutions can be characterized by the coefficients of the original problem. Thus the appropriate local weight functions can be constructed simply, and implementation is a straightforward modification of the original $L^{2}$ least squares functional. And while the theory is developed in weighted Sobolev spaces, practical implementation of the approach may be achieved with standard finite element tools. 
Nonweighted functional results

\begin{tabular}{|c|cc|cc|cc|}
\hline$N$ & $G^{1 / 2}$ & rate & $\left\|u-u^{h}\right\|$ & rate & $\left\|u-u^{h}\right\|_{\Omega \backslash B_{\delta}}$ & rate \\
\hline 1800 & 0.317 & - & $1.23 \mathrm{E}-02$ & - & $2.48 \mathrm{E}-03$ & - \\
5000 & 0.192 & 0.99 & $8.94 \mathrm{E}-03$ & 0.62 & $8.70 \mathrm{E}-03$ & 2.05 \\
9800 & 0.138 & 0.99 & $7.34 \mathrm{E}-03$ & 0.58 & $4.61 \mathrm{E}-04$ & 1.89 \\
16200 & 0.107 & 0.99 & $6.34 \mathrm{E}-03$ & 0.58 & $3.04 \mathrm{E}-04$ & 1.66 \\
24200 & 0.0880 & 0.99 & $5.64 \mathrm{E}-03$ & 0.59 & $2.28 \mathrm{E}-04$ & 1.45 \\
39200 & 0.0694 & 0.98 & $4.88 \mathrm{E}-03$ & 0.60 & $1.69 \mathrm{E}-04$ & 1.24 \\
57800 & 0.0574 & 0.98 & $4.34 \mathrm{E}-03$ & 0.61 & $1.37 \mathrm{E}-04$ & 1.10 \\
80000 & 0.0489 & 0.98 & $3.92 \mathrm{E}-03$ & 0.62 & $1.16 \mathrm{E}-04$ & 1.03 \\
\hline Optimal & - & 1 & - & $\approx 1.62$ & - & 2 \\
\hline
\end{tabular}

Weighted functional results, $w_{1}=1.0, w_{2}=0.5$

\begin{tabular}{|c|cc|cc|cc|}
\hline$N$ & $G_{w}^{1 / 2}$ & rate & $\left\|u-u^{h}\right\|$ & rate & $\left\|u-u^{h}\right\|_{\Omega \backslash B_{\delta}}$ & rate \\
\hline 1800 & 0.278 & - & $7.85 \mathrm{E}-03$ & - & $2.70 \mathrm{E}-03$ & - \\
5000 & 0.167 & 1.00 & $3.43 \mathrm{E}-03$ & 1.62 & $9.79 \mathrm{E}-04$ & 1.98 \\
9800 & 0.119 & 1.00 & $1.99 \mathrm{E}-03$ & 1.62 & $5.02 \mathrm{E}-04$ & 1.99 \\
16200 & 0.0928 & 1.00 & $1.32 \mathrm{E}-03$ & 1.62 & $3.05 \mathrm{E}-04$ & 1.98 \\
24200 & 0.0759 & 1.00 & $9.54 \mathrm{E}-04$ & 1.63 & $2.05 \mathrm{E}-04$ & 1.98 \\
39200 & 0.0597 & 1.00 & $6.44 \mathrm{E}-04$ & 1.63 & $1.27 \mathrm{E}-04$ & 1.98 \\
57800 & 0.0491 & 1.00 & $4.68 \mathrm{E}-04$ & 1.64 & $8.68 \mathrm{E}-05$ & 1.97 \\
80000 & 0.0418 & 1.00 & $3.58 \mathrm{E}-04$ & 1.64 & $6.31 \mathrm{E}-05$ & 1.96 \\
\hline Optimal & - & 1 & - & $\approx 1.62$ & - & 2 \\
\hline
\end{tabular}

TABLE 4.4. Convergence of approximations for nonweighted least squares functional vs. using a weighted functional for $\beta=0.5$, $\alpha=-0.25$ and $\lambda=0.62$.

\begin{tabular}{|c|cc|cc|}
\hline$N$ & $E_{h}$ & rate & $G_{w}^{1 / 2}$ & rate \\
\hline 50 & 0.253 & - & 1.19 & - \\
200 & 0.141 & 0.84 & 0.643 & 0.89 \\
968 & 0.0616 & 1.05 & 0.300 & 0.97 \\
2592 & 0.0365 & 1.06 & 0.186 & 0.98 \\
3872 & 0.0294 & 1.07 & 0.153 & 0.98 \\
10952 & 0.0168 & 1.08 & 0.0918 & 0.98 \\
14792 & 0.0143 & 1.08 & 0.0793 & 0.98 \\
\hline
\end{tabular}

TABLE 4.5. Weighted norm and weighted functional convergence for $\beta=0, \alpha=-1$, and $\lambda=0.5$. 


\section{Acknowledgement}

The authors would like to thank the anonymous referees for their insightful suggestions, especially with respect to the approximation properties of RT spaces.

\section{REFERENCES}

1. G. Acosta, T. Apel, R.G. Durán, and A.L. Lombardi, Error estimates for Raviart-Thomas interpolation of any order on anisotropic tetrahedra, Math. Comp. 80 (2011), No. 273, 141163. MR2728975 (2011m:65262)

2. R. Adams and J. Fournier, Sobolev spaces, second ed., Academic Press, 2003. MR2424078 (2009e:46025)

3. D. Arroyo, A. Bespalov, and N. Heuer, On the finite element method for elliptic problems with degenerate and singular coefficients, Math. Comp. 76 (2007), no. 258, 509-537. MR2291826 (2008e:65336)

4. C. Bacuta, V. Nistor, and L. Zikatanov, Improving the rate of convergence of high-order finite elements on polyhedra $i$ : a priori estimates, Numer. Funct. Anal. Optim. 26 (2005), no. 6, 613-639. MR2187917 (2006i:35036)

5. P.B. Bochev and M.D. Gunzburger, Finite element methods of least-squares type, SIAM Rev. 40 (1998), 789-837. MR.1659689 (99k:65104)

6. D. Boffi, F. Brezzi, L.F. Demkowicz, R.G. Duran, R.S. Falk, and M. Fortin, Mixed finite elements, compatibility conditions, and applications, Lecture Notes in Mathematics, vol. 1939, Springer-Verlag, 2008, Edited by D. Boffi and L. Gastaldi. MR2459075 (2010h:65219)

7. D. Braess, Finite elements: Theory, fast solvers and applications in solid mechanics, Cambridge, 2001. MR1827293(2001k:65002)

8. J. Bramble and J. Pasciak, New estimates for multilevel algorithms including the V-cycle, Math. Comp. 60 (1993), 447-471. MR.1176705 (94a:65064)

9. S.C. Brenner and L.R. Scott, The mathematical theory of finite element methods, SpringerVerlag, 1994. MR1278258 (95f:65001)

10. F. Brezzi and M. Fortin, Mixed and hybrid finite element methods, Springer-Verlag, 1991. MR.1115205(92d:65187)

11. Z. Cai, R. Lazarov, T.A. Manteuffel, and S.F. McCormick, First-order system least squares for second-order partial differential equations: Part I, SIAM J. Numer. Anal. 31 (1994), no. 6, 1785-1799. MR.1302685 (95i:65133)

12. Z. Cai, T.A. Manteuffel, and S.F. McCormick, First-order system least squares for secondorder partial differential equations: Part II, SIAM J. Numer. Anal. 34 (1997), no. 2, 425-454. MR1442921 (98m:65039)

13. Z. Cai and C.R. Westphal, A weighted H(div) least-squares method for second-order elliptic problems, SIAM J. Numer. Anal. 46 (2008), no. 3, 1640-1651. MR.2391010 (2008m:65314)

14. M.D. Gunzburger and P.B. Bochev, Least-squares finite element methods, Springer, 2009. MR2490235 (2010b:65004)

15. Dennis Jespersen, Ritz-Galerkin methods for singular boundary value problems, SIAM J. Numer. Anal. 15 (1978), no. 4, 813-834. MR0488786 (58:8296)

16. R.C. Kirby, From functional analysis to iterative methods, SIAM Review 52 (2010), no. 2, 269-293. MR2646804 (2011j:65279)

17. V.A. Kozlov, V.G. Maz'ya, and J. Rossmann, Elliptic boundary vaule problems in domains with point singularities, vol. 52, American Mathematical Society, 1997. MR.1469972 (98f:35038)

18. L.D. Landau and E.M. Lifshitz, Quantum mechanics: Non-relativistic theory, Pergamon Press, 1958. MR0400931 (53:4761)

19. E. Lee, T.A. Manteuffel, and C.R. Westphal, Weighted-norm first-order system least squares (FOSLS) for problems with corner singularities, SIAM J. Numer. Anal. 44 (2006), no. 5, 1974-1996. MR2263037(2008a:65221)

20. __ Weighted-norm first-order system least squares (FOSLS) for div/curl systems with three dimensional edge singularities, SIAM J. Numer. Anal. 46 (2008), no. 3, 1619-1639. MR2391009 (2009c:65316)

21. H. Li, A-priori analysis and the finite element method for a class of degenerate elliptic equations, Math. Comp. 78 (2009), no. 266, 713-37. MR2476557 (2010b:35163) 
22. Finite element analysis for the axisymmetric laplace operator on polygonal domains, J. Comput. Appl. Math. 235 (2011), 5155-76. MR2817318

23. A. Lunardi, G. Metafune, and D. Pallara, Dirichlet boundary conditions for elliptic operators with unbounded drift, Journal: Proc. Amer. Math. Soc. (2005), no. 133, 2625-2635. MR2146208(2006e:35140)

24. G. Metafune, D. Pallara, J. Pruss, and R. Schnaubelt, $L^{p}$-theory for elliptic operators on $R^{d}$ with singular coefficients, Z. Anal. Anwendungen 24 (2005), no. 3, 497-521. MR2208037 (2006k:35163)

25. P.J. Rabier, Elliptic problems on $R^{N}$ with unbounded coefficients in classical Sobolev spaces, Math. Z. 249 (2005), no. 1, 1-30. MR2106968 (2005h:35054)

26. J. Ruge and K. Stüben, Efficient solution of finite difference and finite element equations, Multigrid methods for integral and differential equations (Bristol, 1983), Inst. Math. Appl. Conf. Ser. New Ser., vol. 3, Oxford Univ. Press, New York, 1985, pp. 169-212. MR849374 (87i:65047)

27. U. Trottenberg, C. Oosterlee, and A. Schüller, Multigrid, Academic Press, 2001. MR.1807961 (2002b:65002)

28. H. Wu and D.W.L. Sprung, Inverse-square potential and the quantum vortex, Phy. Rev. A 49 (1994), no. 6, 4305-11.

Department of Mathematics, Tufts University, Medford, Massachusetts 02155

E-mail address: bidwell.stephen@gmail.com

Department of Mathematics, Binghamton University, Binghamton, New York 139026000

E-mail address: hassell.matthew@gmail.com

Department of Mathematics and Computer Science, Wabash College, P.O. Box 352, Crawfordsville, Indiana 47933

E-mail address: westphac@wabash.edu 